CERN-TH/99-358

astro-ph/9911440

\title{
Particles and Cosmology: Learning from Cosmic Rays
}

\author{
John Ellis* \\ * Theoretical Physics Division, CERN \\ CH - 1211 Geneva 23 \\ Contribution to the Proceedings of the 26th International Cosmic-Ray Conference, \\ Salt Lake City, August 1999
}

\begin{abstract}
The density budget of the Universe is reviewed, and then specific particle candidates for non-bayonic dark matter are introduced, with emphasis on the relevance of cosmic-ray physics. The sizes of the neutrino masses indicated by recent atmospheric and solar neutrino experiments may be too small to contribute much hot dark matter. My favoured candidate for the dominant cold dark matter is the lightest supersymmetric particle, which probably weighs between about $50 \mathrm{GeV}$ and about $600 \mathrm{GeV}$. Strategies to search for it via cosmic rays due to annihilations in the halo, Sun and Earth, or via direct scattering experiments, are mentioned. Possible superheavy relic particles are also discussed, in particular metastable string- or $M$-theory cryptons, that may be responsible for the ultra-high-energy cosmic rays. Finally, it is speculated that a non-zero contribution to the cosmological vacuum energy might result from incomplete relaxation of the quantum-gravitational vacuum.
\end{abstract}

\section{DENSITY BUDGET OF THE UNIVERSE}

As you know, the Universe becomes almost homogeneous and isotropic, when viewed on a sufficiently large scale. This suggests very strongly that it may be described approximately by a Robertson-Walker-Friedmann metric. The crucial parameters describing the expansion of the Universe are then its density $\rho$ and the curvature of the Universe; $k=0,+1$ or -1 for a critical, closed or open Universe, respectively. If $k=0$, the density must equal the critical density $\rho_{c} \sim 2 h^{2} \times 10^{-29}$ 
$\mathrm{g} / \mathrm{cm}^{3}$, where $h$ is the present Hubble expansion rate in units of $100 \mathrm{~km} / \mathrm{s} / \mathrm{Mpc}$. Much of the subsequent cosmological discussion is phrased in terms of the density budget of the Universe, expressed as contributions relative to the critical density: $\Omega_{i} \equiv \rho_{i} / \rho_{c}$.

$\Omega_{\text {tot }}$ : Inflation suggests that this is practically indistinguishable from unity: $\Omega_{\text {tot }}=1 \pm \mathcal{O}\left(10^{-4}\right)[1]$, although there are some models that predict $\Omega_{t o t}<1[2]$. However, the data on the small anisotropies in the cosmic Microwave Background (CMB) [3] support the inflationary suggestion that $\Omega_{t o t} \sim 1$ and $\Omega_{k} \sim 0$, as summarized in Fig. 1 [4].

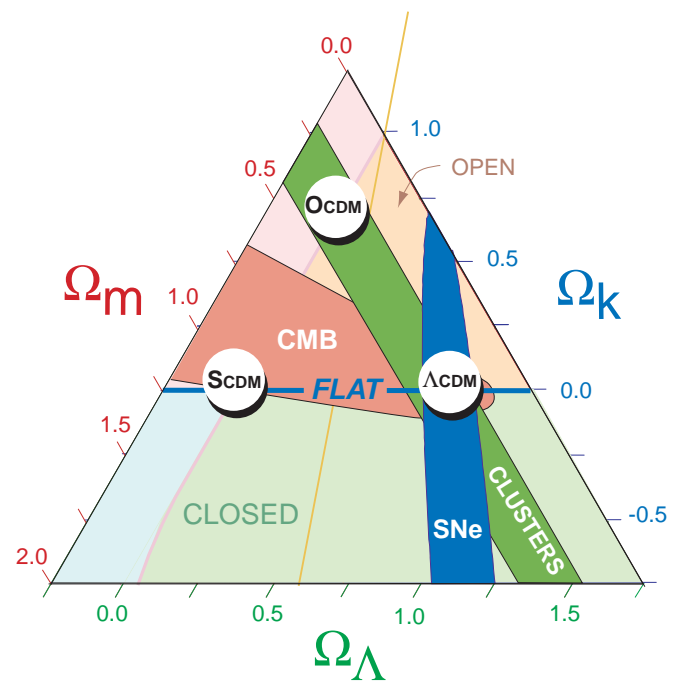

FIGURE 1. Compilation [4] of constraints on contributions to the cosmological energy density, as provided by the cosmic microwave background (CMB), cluster data and high-redshift supernovae. Concordance appears for the model $\Lambda_{C D M}$ with cosmological vacuum energy, but not for standard cold dark matter $S_{C D M}$ or for an open dark matter model $O_{C D M}$.

$\Omega_{b}$ : Measurements of the $D / H$ ratio in high-redshift Lyman- $\alpha$ clouds [5] correspond to

$$
\frac{D}{H}=(3.3 \pm 0.3) \times 10^{-5}
$$

If this is indeed the correct primordial $D / H$ ratio, Big-Bang nucleosynthesis calculations suggest that [5]

$$
\frac{n_{B}}{s}=(5.1 \pm 0.3) \times 10^{-10}
$$

corresponding to 


$$
\Omega_{B} h^{2}=0.019 \pm 0.001
$$

Using the currently favoured range $h=0.65 \pm 0.10$, we see from (20) that $\Omega_{b} \lesssim 0.08$, which is insufficient to explain all the matter density in the following paragraph.

$\Omega_{m}$ : The cluster measurements $(M / L$ ratio, present and past abundances, cluster dynamics and the baryon fractions inferred from $X$-ray measurements) all suggest [6]

$$
\Omega_{m} \sim 0.2 \text { to } 0.3
$$

as also seen in Fig. 1. Moreover, the combination of CMB measurements and high-redshift supernovae [7] also support independently such a value for $\Omega_{m}$.

$\Omega_{C D M}$ : The theory [4] of large-scale structure formation strongly suggests that most of $\Omega_{m}$ is cold dark matter, so that

$$
\Omega_{C D M} \sim \Omega_{m}
$$

as perhaps provided by the supersymmetric particles discussed later.

$\Omega_{H D M}$ : The theory [4] of structure formation also suggests that the density of hot dark matter $\Omega_{H D M} \ll \Omega_{C D M}$. The present and prospective sensitivities of cosmological data to $m_{\nu}$ are shown in Fig. 2 [8]. So far, $m_{\nu} \gtrsim 3 \mathrm{eV}$ is excluded by the available upper limit on the density of hot dark matter, whereas the possible comparison of future data on large-scale structure and the CMB are thought to be sensitive to $m_{\nu} \gtrsim 0.3 \mathrm{eV}$. This is somewhat above the range $m_{\nu} \sim 0.1$ to 0.03 $\mathrm{eV}$ favoured by the atmospheric neutrino data, but one should not abandon hope of detecting neutrino masses astrophysically [8]. As discussed in Section 2, the indications of neutrino masses from atmospheric and solar neutrino data can most easily be explained by light neutrinos: $m_{\nu_{i}}<0.1 \mathrm{eV}$, which would make only a small contribution to $\Omega_{t o t}$.

$\Omega_{\Lambda}$ : If one follows the inflationary path supported by the CMB [3], so that $\Omega_{\text {tot }} \sim 1$, and takes at face value the suggestions from cluster measurements that $\Omega_{m} \sim 0.3$, then the largest fraction of the energy density of the Universe may be provided by vacuum energy: $\Omega_{\Lambda} \sim 0.7$. This scenario is supported by the recent high-redshift supernova data [7] shown in Fig. 3, which suggest that $\Omega_{\Lambda}-\Omega_{m} \sim 0.4$. Combining this estimate with the suggestion of inflation that $\Omega_{\text {tot }}=\Omega_{m}+\Omega_{\Lambda} \simeq 1$, one recovers independently the preference for $\Omega_{m} \sim 0.3, \Omega_{\Lambda} \sim 0.7$.

A remarkably consistent picture of the density budget of the Universe may be emerging:

$$
\Omega_{\text {tot }} \simeq 1=\Omega_{m}+\Omega_{\Lambda}: \Omega_{m} \sim 0.3, \Omega_{\Lambda} \sim 0.7
$$




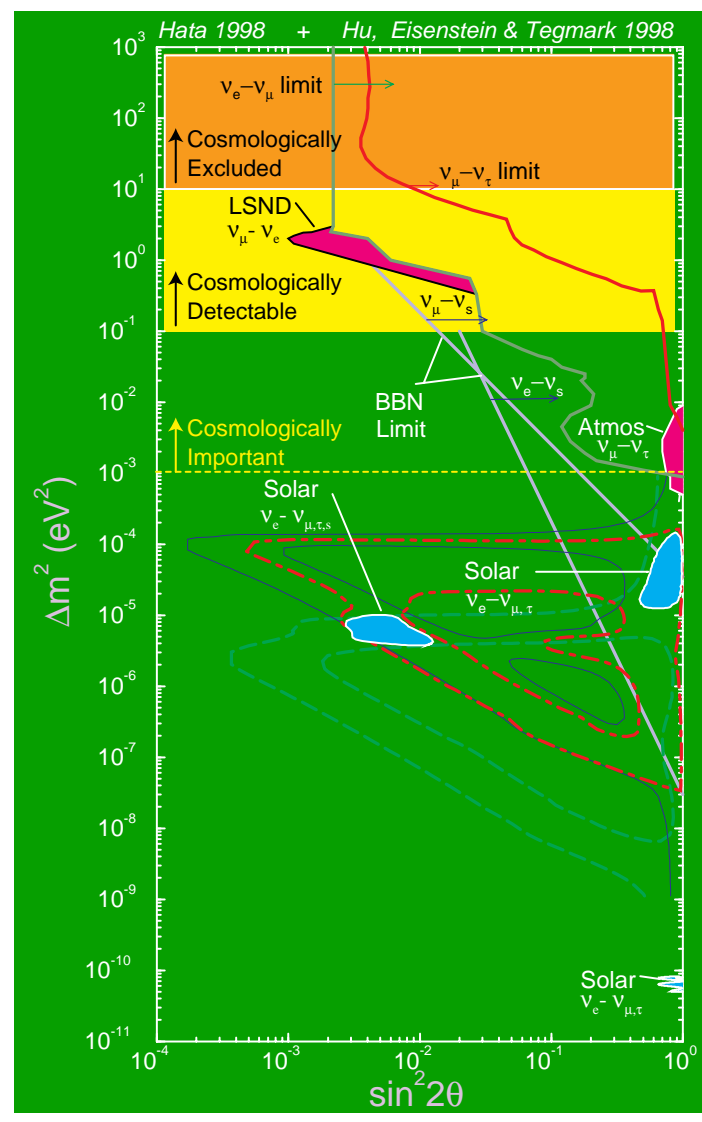

FIGURE 2. Compilation [8] of indications on neutrino mass-squared differences $\Delta m^{2}$ and mixing angles $\theta$ from oscillation experiments, compared with cosmological sensitivities to neutrino masses.

where

$$
\Omega_{m}=\Omega_{C D M}+\Omega_{\nu}+\Omega_{b}
$$

with

$$
\Omega_{b}<0.1, \quad \Omega_{\nu} \ll \Omega_{C D M} \simeq \Omega_{m}
$$

It remains to be seen whether future data confirm this picture. For the moment, let us examine particle candidates for $\Omega_{H D M}$ and $\Omega_{C D M}$, emphasizing their cosmic-ray manifestations. 


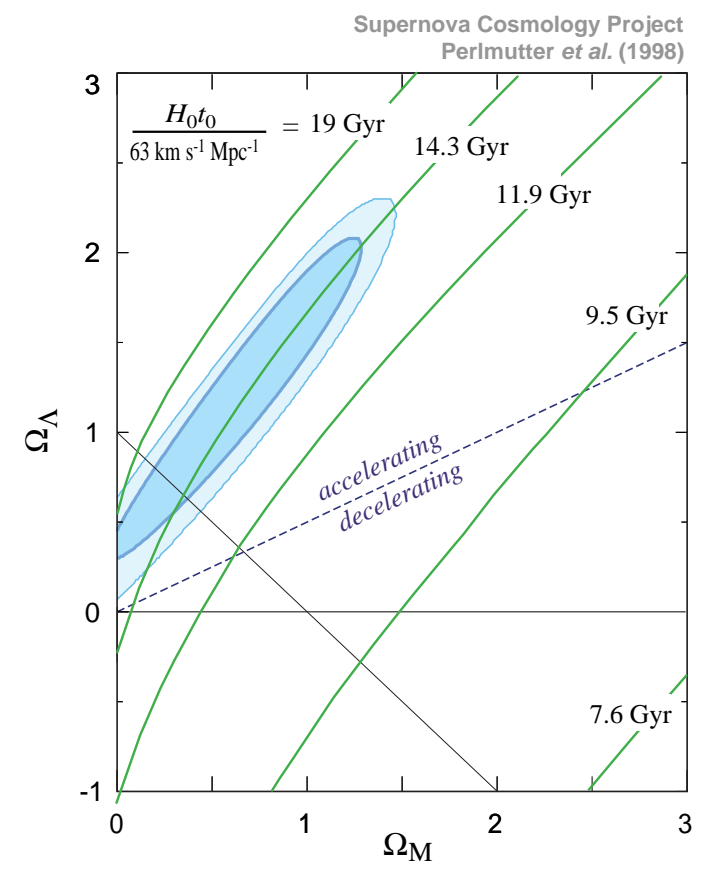

Best fit age of universe: $t_{0}=14.5 \pm 1(0.63 / h)$ Gyr

Best fit in flat universe: $t_{0}=14.9 \pm 1(0.63 / h) \mathrm{Gyr}$

www-supernova.LBL.gov

FIGURE 3. Constraint on $\Omega_{\Lambda}, \Omega_{M}$ from one set of high-redshift supernova data [7].

\section{NEUTRINO MASSES}

If these are non-zero, laboratory experiments tell us that they must be much smaller than those of the corresponding charged leptons [9]:

$$
m_{\nu_{e}} \lesssim 2.5 \mathrm{eV}, \quad m_{\nu_{\mu}} \lesssim 160 \mathrm{keV}, \quad m_{\nu_{\tau}} \lesssim 15 \mathrm{eV},
$$

so one might think naively that they should vanish entirely. However, theorists believe that particle masses can be strictly zero only if there is a corresponding conserved charge associated with an exact gauge symmetry, which is not the case for lepton number. Indeed, non-zero neutrino masses appear generically in Grand Unified Theories (GUTs) [10]. However, it is not even necessary to postulate new particles to get $m_{\nu} \neq 0$. These could be generated by a non-renormalizable interaction among Standard Model particles [11]: 


$$
\frac{\left(\nu_{L} H\right)\left(\nu_{L} H\right)}{M}
$$

where $M \gg m_{W}$ is some new, heavy mass scale. The most plausible guess, though, is that this heavy mass is that of some heavy particle, perhaps a right-handed neutrino $\nu_{R}$ with mass $M \sim M_{G U T}$.

In this case, one expects to find the characteristic see-saw [12] form of neutrino mass matrix:

$$
\left(\nu_{L}, \nu_{R}\right) \quad\left(\begin{array}{cc}
0 & m \\
m & M
\end{array}\right) \quad\left(\begin{array}{c}
\nu_{L} \\
\nu_{R}
\end{array}\right)
$$

where the off-diagonal matrix entries in (11) break $\mathrm{SU}(2)$ and have the form of Dirac mass terms, so that one expects $m=\mathcal{O}\left(m_{\ell, q}\right)$. Diagonalizing (11), one finds generically a light neutrino mass

$$
m_{\nu} \simeq \frac{m^{2}}{M}
$$

Choosing representative numbers $m \sim 10 \mathrm{GeV}, m_{\nu} \sim 10^{-2} \mathrm{eV}$, one finds $M \sim 10^{13}$ $\mathrm{GeV}$, in the general ballpark of the grand unification scale.

As you know, data on both solar and atmospheric neutrinos favour neutrino oscillations associated with neutrino mass differences: $\nu_{e} \rightarrow \nu_{x}$ in the solar case and $\nu_{\mu} \rightarrow \nu_{x}$ in the atmospheric case. (Both of these can be regarded as cosmic-ray phenomena!) There are three possible interpretations of the solar-neutrino data: vacuum oscillations with $\Delta m^{2} \sim 10^{-10} \mathrm{eV}^{2}$ and large mixing, and matter-enhanced MSW oscillations with $\Delta m^{2} \sim 10^{-5} \mathrm{eV}^{2}$ and either large or small mixing, as seen in Fig. 4 [13]. There is no hint what (combination of) other flavours the $\nu_{e}$ might be oscillating into. In the atmospheric case, $\Delta m^{2} \sim(2$ to 6$) \times 10^{-3} \mathrm{eV}^{2}$ and large mixing are required. The Super-Kamiokande and Chooz data both exclude $\nu_{\mu} \rightarrow \nu_{e}$ dominance, and zenith-angle distributions in the Super-Kamiokande data favour $\nu_{\mu} \rightarrow \nu_{\tau}$ over $\nu_{\mu}$ oscillations into sterile neutrinos $\nu_{s}$.

The past year has witnessed many theoretical studies of neutrino masses [14], of which I now pick out just a few key features:

Other light neutrinos? We know from the LEP neutrino-counting constraint [15], that any additional neutrinos must be sterile $\nu_{s}$, with no electroweak interactions or quantum numbers. But if so, what is to prevent them from acquiring large masses: $M_{s} \nu_{s} \nu_{s}$ with $M_{s} \gg m_{W}$, as for the $\nu_{R}$ discussed above? In the absence of some new theoretical superstructure, this is an important objection to simply postulating light $\nu_{s}$ or $\nu_{R}$. 

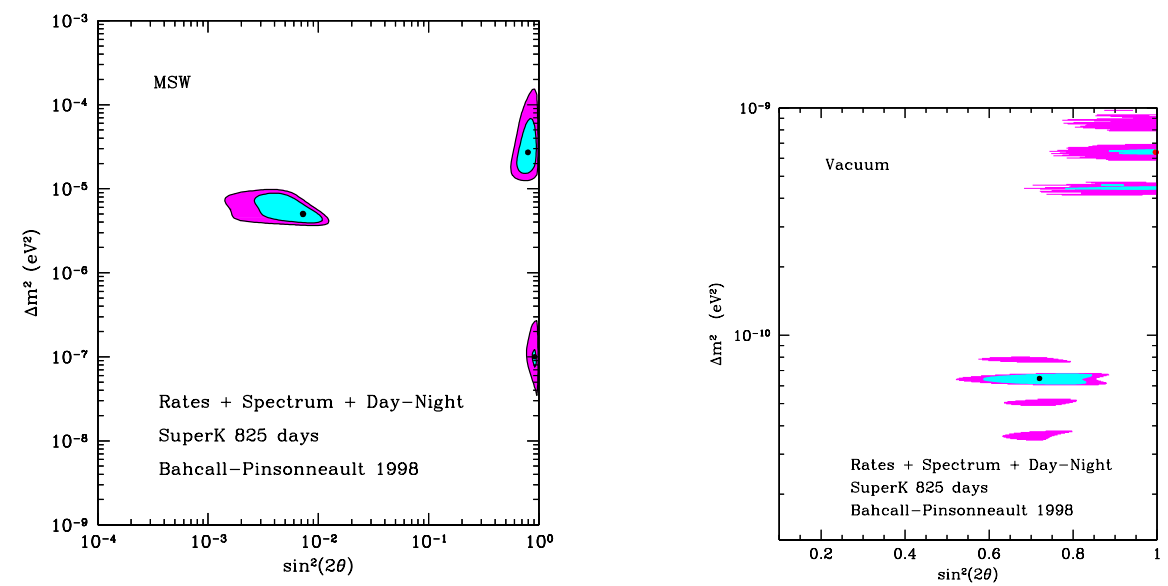

FIGURE 4. Regions of $\Delta m^{2}$ and $\sin ^{2} 2 \theta$ for $\nu-e \rightarrow \nu_{x}$ oscillations favoured by a global analysis of solar neutrino data [13].

Majorana masses? Most theorists expect the light neutrinos to be essentially pure $\nu_{L}$, with only a small admixture $\mathcal{O}(m / M)$ of $\nu_{R}$. In this case, one expects the dominant effective neutrino mass term to be of Majorana type $m_{e f f} \nu_{L} \nu_{L}$, as given by (10) or (12).

Large mixing? Small neutrino mixing used perhaps to be favoured, by analogy with the Cabibbo-Kobayashi-Maskawa mixing of quarks. However, theorists now realize that this is by no means necessary. For one thing, the off-diagonal entries in (now considered as a $3 \times 3$ matrix) (12) need not be $\propto m_{q}$ or $m_{\ell}$ [16]. Moreover, even if $m \propto m_{\ell}$, we have no independent evidence that mixing is small in the lepton sector. Finally, even if $m$ were to be approximately diagonal in the same flavour basis as the charged leptons $e, \mu, \tau$, why should this also be the same case for the heavy Majorana matrix $M[16]$ ?

Could neutrinos be degenerate? Are masses $\bar{m} \gtrsim 2 \mathrm{eV}$ and close to the direct and astrophysical limits allowed [17]? Any such scenario would need to respect the stringent constraint imposed by the absence of $\beta \beta_{0 \nu}$ decay [18]:

$$
<m_{\nu}>_{e} \simeq \bar{m}\left|c_{12}^{2} c_{13}^{2} e^{i \alpha}+s_{12}^{2} c_{13}^{2} e^{i \beta}+s_{13}^{2}\right| \lesssim 0.2 \mathrm{eV}
$$

In view of the upper limit on $\nu_{\mu}-\nu_{e}$ mixing from the Chooz experiment [19], let us neglect provisionally the last term in (13). In this case, there must be a cancellation between the first two terms, requiring $\alpha \simeq \beta+\pi$, and

$$
c_{12}^{2}-s_{12}^{2}=\cos 2 \theta_{12} \lesssim 0.1 \Rightarrow \sin ^{2} 2 \theta_{12} \gtrsim 0.99
$$


Thus maximal $\nu_{e}-\nu_{\mu}$ mixing is necessary if the neutrinos are heavy and degenerate. This certainly excludes the small-mixing-angle MSW solution and possibly even the large-mixing-angle MSW solution, since this is not compatible with $\sin ^{2} 2 \theta=1$ (which would yield a constant energy-independent suppression of the solar neutrino flux), and global fits typically indicate that $\sin ^{2} \theta_{12} \lesssim 0.97$, as seen in Fig. 5 [20]. Global fits before the new Super-Kamiokande data on the energy spectrum indi-

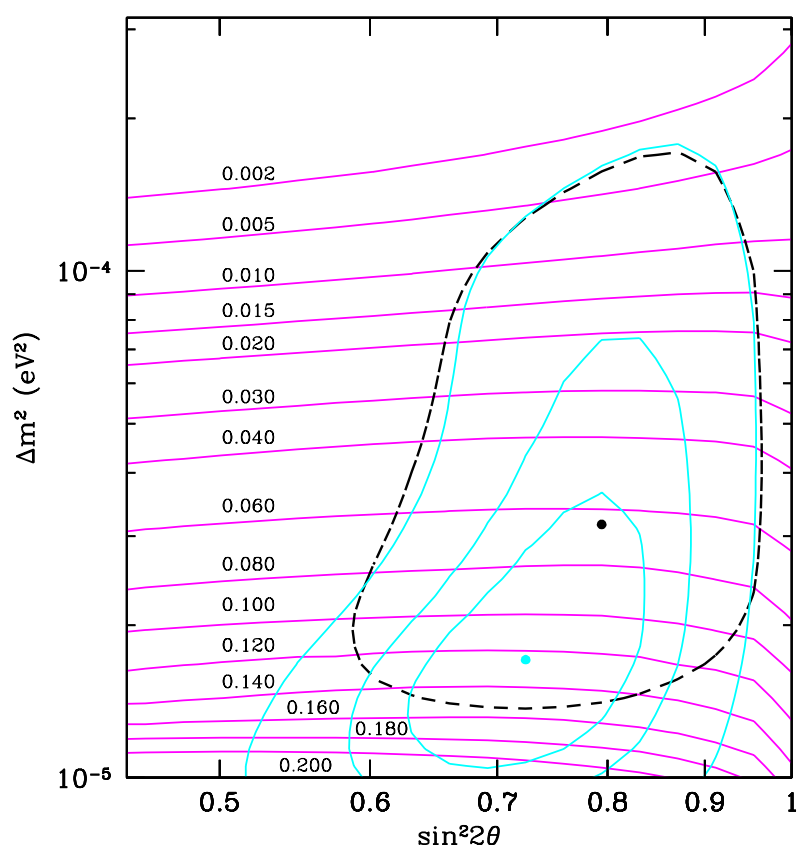

FIGURE 5. Preferred region of $\sin ^{2} \theta$ and $\Delta m^{2}$ for the large-mixing-angle MSW solution to the solar neutrino problem, both with (dashed contours) and without (grey contours) the measured day-night asymmetry: note that $\sin ^{2} \theta<0.97$ [20].

cated that $\sin ^{2} 2 \theta \sim 1$ was possible for vacuum-oscillation solutions. However, the new Super-Kamiokande analysis of the energy spectrum now indicates [21] that, if there is any consistent vacuum-oscillation solution at all, it may well have $\sin ^{2} 2 \theta$ considerably below 1 , providing another potential nail in the coffin of degenerate neutrinos.

The vacuum-oscillation solution would require, moreover, extreme degeneracy: $\Delta m \sim 10^{-10} \bar{m}$, which is impossible to reconcile with a simple calculation of neutrino mass renormalization in models with degenerate masses at the $m_{\nu_{R}}$ scale [17]. Mass-renormalization effects also endanger the large-angle MSW solution (which would require $\Delta m \sim 10^{-4} \bar{m}$ ), and, in the context of bimaximal mixing models, also generate unacceptable values of the neutrino mixing angles. These renormalization problems may not be insurmountable [22], but they do raise non-trivial issues that must be addressed in models [23] of (near-)degenerate neutrino masses. 
Our provisional conclusion is that $m_{\nu_{i}} \ll 2 \mathrm{eV}$, with the most likely case being $m_{\nu_{i}} \lesssim \sqrt{\Delta m_{\text {atmo }}^{2}} \sim 0.06 \mathrm{eV}$, too small to be of much interest for hot dark matter.

\section{THE LIGHTEST SUPERSYMMETRIC PARTICLE}

The motivation for supersymmetry at an accessible energy is provided by the gauge hierarchy problem [24], namely that of understanding why $m_{W} \ll m_{P}$, the only candidate for a fundamental mass scale in physics. Alternatively and equivalently, one may ask why $G_{F} \sim g^{2} / m_{W}^{2} \gg G_{N}=1 / m_{P}^{2}$, where $M_{P}$ is the Planck mass, expected to be the fundamental gravitational mass scale. Or one may ask why the Coulomb potential inside an atom is so much larger than the Newton potential, which is equivalent to why $e^{2}=\mathcal{O}(1) \gg m_{p} m_{e} / m_{P}^{2}$, where $m_{p, e}$ are the proton and electron masses.

One might think it would be sufficient to choose the bare mass parameters: $m_{W} \ll m_{P}$. However, one must then contend with quantum corrections, which are quadratically divergent:

$$
\delta m_{H, W}^{2}=\mathcal{O}\left(\frac{\alpha}{\pi}\right) \Lambda^{2}
$$

These are much larger than $m_{W}$, if the cutoff $\Lambda$ representing the appearance of new physics is taken to be $\mathcal{O}\left(m_{P}\right)$. This means that one must fine-tune the bare mass parameter so that it is almost exactly cancelled by the quantum correction (15) in order to obtain a small physical value of $m_{W}$. This seems unnatural, and the alternative is to introduce new physics at the $\mathrm{TeV}$ scale, so that the correction (15) is naturally small.

At one stage, it was proposed that this new physics might correspond to the Higgs boson being composite [25]. However, calculable scenarios of this type are inconsistent with the precision electroweak data from LEP and elsewhere. The alternative is to postulate approximate supersymmetry [26], whose pairs of bosons and fermions produce naturally cancelling quantum corrections:

$$
\delta m_{W}^{2}=\mathcal{O}\left(\frac{\alpha}{\pi}\right)\left|m_{B}^{2}-m_{F}^{2}\right|
$$

that are naturally small: $\delta m_{W}^{2} \lesssim m_{W}^{2}$ if

$$
\left|m_{B}^{2}-m_{F}^{2}\right| \lesssim 1 \mathrm{TeV}^{2} .
$$

There are many other possible motivations for supersymmetry, some of which are discussed below, but this is the only one that gives reason to expect that it might 
be accessible to the current generation of accelerators and in the range expected for a cold dark matter particle.

The minimal supersymmetric extension of the Standard Model (MSSM) [27] has the same gauge interactions as the Standard Model, and the Yukawa interactions are very similar:

$$
\lambda_{d} Q D^{c} H+\lambda_{\ell} L E^{c} H+\lambda_{u} Q U^{c} \bar{H}+\mu \bar{H} H
$$

where the capital letters denote supermultiplets with the same quantum numbers as the left-handed fermions of the Standard Model. The couplings $\lambda_{d, \ell, u}$ give masses to down quarks, leptons and up quarks respectively, via distinct Higgs fields $H$ and $\bar{H}$, which are required in order to cancel triangle anomalies. The new parameter in (18) is the bilinear coupling $\mu$ between these Higgs fields, that plays a significant rôle in the description of the lightest supersymmetric particle, as we see below. The gauge quantum numbers do not forbid the appearance of additional couplings [28]

$$
\lambda L L E^{c}+\lambda^{\prime} L Q D^{c}+\lambda U^{c} D^{c} D^{c}
$$

but these violate lepton or baryon number, and we assume they are absent. One significant aspect of the MSSM is that the quartic scalar interactions are determined, leading to important constraints on the Higgs mass, as we also see below.

Supersymmetry must be broken, since supersymmetric partner particles do not have identical masses, and this is usually parametrized by scalar mass parameters $m_{0_{i}}^{2}\left|\phi_{i}\right|^{2}$, gaugino masses $\frac{1}{2} M_{a} \tilde{V}_{a} \cdot \tilde{V}_{a}$ and trilinear scalar couplings $A_{i j k} \lambda_{i j k} \phi_{i} \phi_{j} \phi_{k}$. These are commonly supposed to be inputs from some high-energy physics such as supergravity or string theory. It is often hypothesized that these inputs are universal: $m_{0_{i}} \equiv m_{0}, M_{a} \equiv m_{1 / 2}, A_{i j k} \equiv A$, but these assumptions are not strongly motivated by any fundamental theory. The physical sparticle mass parameters are then renormalized in a calculable way:

$$
m_{0_{i}}^{2}=m_{0}^{2}+C_{i} m_{1 / 2}^{2}, \quad M_{a}=\left(\frac{\alpha_{a}}{\alpha_{G U T}}\right) m_{1 / 2}
$$

where the $C_{i}$ are calculable coefficients [29] and MSSM phenomenology is then parametrized by $\mu, m_{0}, m_{1 / 2}, A$ and $\tan \beta$ (the ratio of Higgs v.e.v.'s).

Precision electroweak data from LEP and elsewhere provide two qualitative indications in favour of supersymmetry. One is that the inferred magnitude of quantum corrections favour a relatively light Higgs boson [15]

$$
m_{h} \lesssim 200 \mathrm{GeV}
$$

which is highly consistent with the value predicted in the MSSM: $m_{h} \lesssim 150 \mathrm{GeV}$ [30] as a result of the constrained quartic couplings. (On the other hand, composite 
Higgs models predicted an effective Higgs mass $\gtrsim 1 \mathrm{TeV}$ and other unseen quantum corrections.) The other indication in favour of low-energy supersymmetry is provided by measurements of the gauge couplings at LEP, that correspond to $\sin ^{2} \theta_{W} \simeq 0.231$ in agreement with the predictions of supersymmetric GUTs with sparticles weighing about $1 \mathrm{TeV}$, but in disagreement with non-supersymmetric GUTs that predict $\sin ^{2} \theta_{W} \sim 0.21$ to 0.22 [31]. Neither of these arguments provides an accurate estimate of the sparticle mass scales, however, since they are both only logarithmically sensitive to $m_{0}$ and/or $m_{1 / 2}$.

The lightest supersymmetric particle (LSP) is expected to be stable in the MSSM, and hence should be present in the Universe today as a cosmological relic from the Big Bang [32,33]. This is a consequence of a multiplicatively-conserved quantum number called $R$ parity, which is related to baryon number, lepton number and spin:

$$
R=(-1)^{3 B+L+2 S}
$$

It is easy to check that $R=+1$ for all Standard Model particles and $R=-1$ for all their supersymmetric partners. The interactions (18) conserve $R$, whilst those in (19) would violate $R$, in contrast to a Majorana neutrino mass term or the other interactions in minimal $S U(5)$ or $S O(10)$ GUTs. There are three important consequences of $R$ conservation: (i) sparticles are always produced in pairs, e.g., $p p \rightarrow \tilde{q} \tilde{g} X, e^{+} e^{-} \rightarrow \tilde{\mu}^{+} \tilde{\mu}^{-}$, (ii) heavier sparticles decay into lighter sparticles, e.g., $\tilde{q} \rightarrow q \tilde{g}, \tilde{\mu} \rightarrow \mu \tilde{\gamma}$, and (iii) the LSP is stable because it has no legal decay mode.

If such a supersymmetric relic particle had either electric charge or strong interactions, it would have condensed along with ordinary baryonic matter during the formation of astrophysical structures, and should be present in the Universe today in anomalous heavy isotopes. These have not been seen in studies of $\mathrm{H}, \mathrm{He}, \mathrm{Be}$, $\mathrm{Li}, \mathrm{O}, \mathrm{C}, \mathrm{Na}, \mathrm{B}$ and $\mathrm{F}$ isotopes at levels ranging from $10^{-11}$ to $10^{-29}$ [34], which are far below the calculated relic abundances from the Big Bang:

$$
\frac{n_{\text {relic }}}{n_{p}} \gtrsim 10^{-6} \text { to } 10^{-10}
$$

for relics with electromagnetic or strong interactions. Except possibly for very heavy relics, one would expect these primordial relic particles to condense into galaxies, stars and planets, along with ordinary bayonic material, and hence show up as an anaomalous heavy isotope of one or more of the elements studied. There would also be a 'cosmic rain' of such relics [35], but this would presumably not be the dominant source of such particles on earth. The conflict with (23) is sufficiently acute that the lightest supersymmetric relic must presumably be electromagnetically neutral and weakly interacting [32]. In particular, I believe that the possibility of a stable gluino can be excluded. This leaves as scandidates for cold dark matter 
a sneutrino $\tilde{\nu}$ with spin 0 , some neutralino mixture of $\tilde{\gamma} / \tilde{H}^{0} / \tilde{Z}$ with spin $1 / 2$, and the gravitino $\tilde{G}$ with spin $3 / 2$.

LEP searches for invisible $Z^{0}$ decays require $m_{\tilde{\nu}} \gtrsim 43 \mathrm{GeV}$ [36], and searches for the interactions of relic particles with nuclei then enforce $m_{\tilde{\nu}} \gtrsim$ few TeV [37], so we exclude this possibility for the LSP. The possibility of a gravitino $\tilde{G}$ LSP has attracted renewed interest recently with the revival of gauge-mediated models of supersymmetry breaking [38], and could constitute warm dark matter if $m_{\tilde{G}} \simeq$ $1 \mathrm{keV}$. In this talk, however, I concentrate on the $\tilde{\gamma} / \tilde{H}^{0} / \tilde{Z}^{0}$ neutralino combination $\chi$, which is the best supersymmetric candidate for cold dark matter.

The neutralinos and charginos may be characterized at the tree level by three parameters: $m_{1 / 2}, \mu$ and $\tan \beta$. The lightest neutralino $\chi$ simplifies in the limit $m_{1 / 2} \rightarrow 0$ where it becomes essentially a pure photino $\tilde{\gamma}$, or $\mu \rightarrow 0$ where it becomes essentially a pure higgsino $\tilde{H}$. These possibilities are excluded, however, by LEP and the FNAL Tevatron collider [36]. From the point of view of astrophysics and cosmology, it is encouraging that there are generic domains of the remaining parameter space where $\Omega_{\chi} h^{2} \simeq 0.1$ to 1 , in particular in regions where $\chi$ is approximately a $U(1)$ gaugino $\tilde{B}$, as seen in Fig. 6 [39].

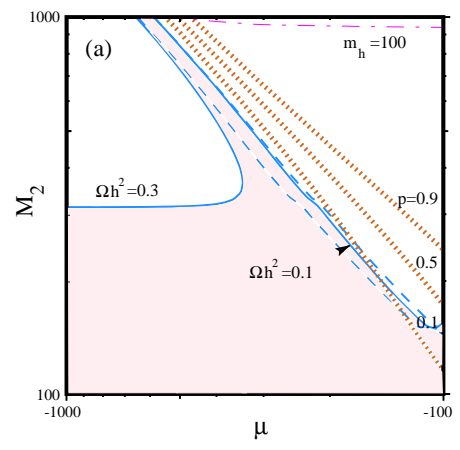

(a)

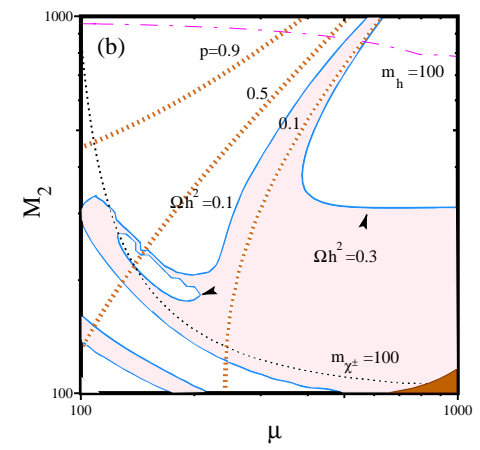

(b)

FIGURE 6. Regions of the $\left(\mu, M_{2}\right)$ plane in which the supersymmetric relic density may lie within the interesting range $0.1 \leq \Omega h^{2} \leq 0.3[14]$.

Purely experimental searches at LEP enforce $m_{\chi} \gtrsim 30 \mathrm{GeV}$ [40]. This bound can be strengthened by making various theoretical assumptions, such as the universality of scalar masses $m_{0_{i}}$, including in the Higgs sector, the cosmological dark matter requirement that $\Omega_{\chi} h^{2} \leq 0.3$ and the astrophysical preference that $\Omega_{\chi} h^{2} \geq 0.1$. Taken together as in Fig. 7, we see that they enforce

$$
m_{\chi} \gtrsim 50 \mathrm{GeV}
$$

Moreover, LEP has already explored almost all the parameter space available for a 

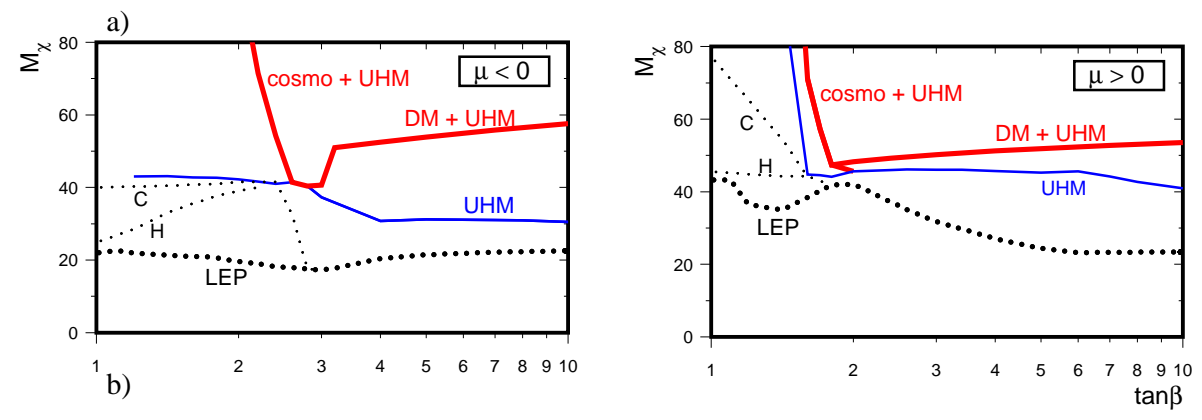

FIGURE 7. Theoretical lower limits on the lightest neutralino mass, obtained by using the unsuccessful Higgs searches $(\mathrm{H})$, the cosmological upper limit on the relic density $(\mathrm{C})$, the assumption that all input scalar masses are universal, including those of the Higgs multiplets (UHM), and combining this with the cosmological upper (cosmo) and astrophysical lower (DM) limits on the cold dark matter density [36].

Higgsino-like LSP, and this possibility will also be thoroughly explored by the full running of LEP [40].

Should one be concerned that no sparticles have yet been seen by either LEP or the FNAL Tevatron collider? One way to quantify this is via the amount of finetuning of the input parameters required to obtain the physical value of $m_{W}$ [41]:

$$
\Delta_{o}=\operatorname{Max}_{i}\left|\frac{a_{i}}{m_{W}} \frac{\partial m_{W}}{\partial a_{i}}\right|
$$

where $a_{i}$ is a generic supergravity input parameter. The LEP exclusions impose [42]

$$
\Delta_{o} \gtrsim 8
$$

Although fine-tuning is a matter of taste, this is perhaps not large enough to be alarming, and could in any case be reduced significantly if a suitable theoretical relation between some input parameters is postulated [42]. Moreover, it is interesting to note that the amount of fine-tuning $\Delta_{o}$ is minimized when $\Omega_{\chi} h^{2} \sim 0.1$ as preferred astrophysically, as seen in Fig. 8 [43]. This means that solving the gauge hierarchy problem naturally leads to a relic neutralino density in the range of interest to astrophysics and cosmology. I am unaware of any analogous argument for other particle dark matter candidates such as the neutrino or the axion.

For certain ranges of the MSSM parameters, our present electroweak vacuum is unstable against the development of vev's for $\tilde{q}$ and $\tilde{l}$ fields, leading to vacua that would break charge and colour conservation. Among the dangerous possibilities are flat directions of the effective potential in which combinations such as 

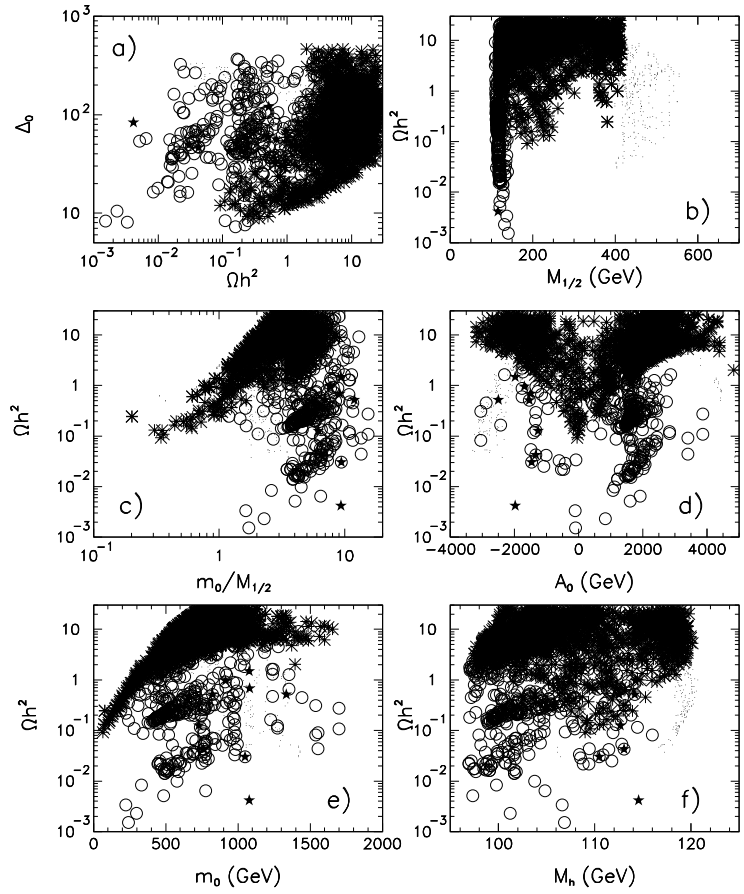

FIGURE 8. The correlation [42] between the fine-tuning price $\Delta_{0}$ and the relic density $\Omega h^{2}$, showing dependences on model parameters.

$L_{i} Q_{3} D_{3}, \quad H_{2} L_{i}, \quad L L E, \quad H_{2} L$ acquire vev's. Avoiding these vacua imposes constraints that depend on the soft supersymmetry breaking parameters: they are weakest for $A \simeq m_{1 / 2}$. Fig. 9 illustrates some of the resulting constraints in the $\left(m_{1 / 2}, m_{0}\right)$ plane, for different values of $\tan \beta$ and signs of $\mu[44]$. We see that they cut out large parts of the plane, particularly for low $m_{0}$. In combination with cosmology, they tend to rule out large values of $m_{1 / 2}$, but this aspect needs to be considered in conjunction with the effects of coannihilation, that are discussed in the next paragraph.

As $m_{\chi}$ increases, the LSP annihilation cross section decreases and hence its relic number and mass density increase. How heavy could the LSP be? Until recently, the limit given was $m_{\chi} \lesssim 300 \mathrm{GeV}$ [45]. However, it has now been pointed out that there are regions of the MSSM parameter space where coannihilations of the $\chi$ with the stau slepton $\tilde{\tau}$ could be important, as also seen in Fig. 9 [46]. These coannihilations would suppress $\Omega_{\chi}$, allowing a heavier neutralino mass, and we now find that $[46]$

$$
m_{\chi} \lesssim 600 \mathrm{GeV}
$$

is possible if we require $\Omega_{\chi} h^{2} \leq 0.3$. In the past, it was thought that all the cosmologically-preferred region of MSSM parameter space could be explored by 

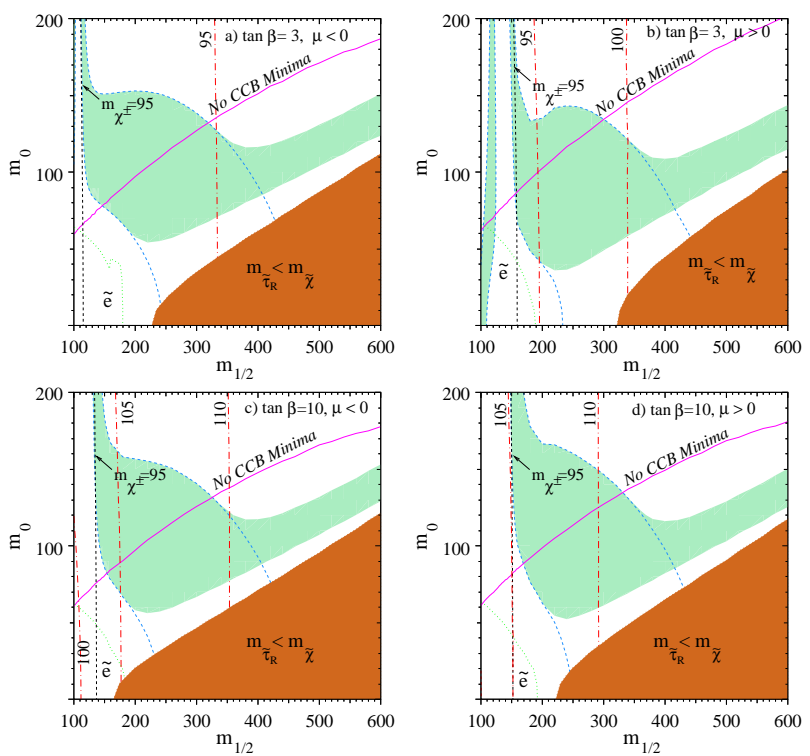

FIGURE 9. The light shaded region is that favoured by calculations of the relic density of LSPs, including coannihilation effects, which are significant on the right sides of the panels [46]. The dark shaded region is excluded because it would have charged dark matter. Also indicated are mass contours of interest to LEP searches, and a potential lower bound on $m_{0}$ obtained by requiring that the true vacuum not break charge and colour conservation (CCB) [44].

the LHC [47], as seen in Fig. 10, but it now seems possible that there may be a delicate region close to the upper bound (27). This point requires further study.

\section{SEARCHES FOR DARK MATTER PARTICLES}

\section{A Annihilation in the Galactic Halo}

One strategy to look for dark matter particles is via their annihilations in the galactic halo; $\chi \chi \rightarrow \ell^{+} \ell^{-}, \bar{q} q \rightarrow \bar{p}, e^{+}, \gamma, \nu$ in the cosmic rays [48]. Figure 11 shows the current measurements of cosmic-ray $\bar{p}$ 's. The lines indicate the secondary flux expected to be produced by primary matter cosmic rays. Some of the earlier measurements were above this conventional expectation, fuelling speculation about possible exotic sources such as $\chi \chi$ annihilation. However, the recent BESS data [49] agree very well with conventional secondary production. There may still be some scope for exotic sources at low energies $E \lesssim 300 \mathrm{MeV}$ or at higher energies $E \gtrsim$ $3 \mathrm{GeV}$, and one of the objectives of the AMS experiment [50] is to explore this possibility. Figure 12 shows that some supersymmetric models are already excluded by the BESS data [49], and indicates how big an opportunity AMS may have. 


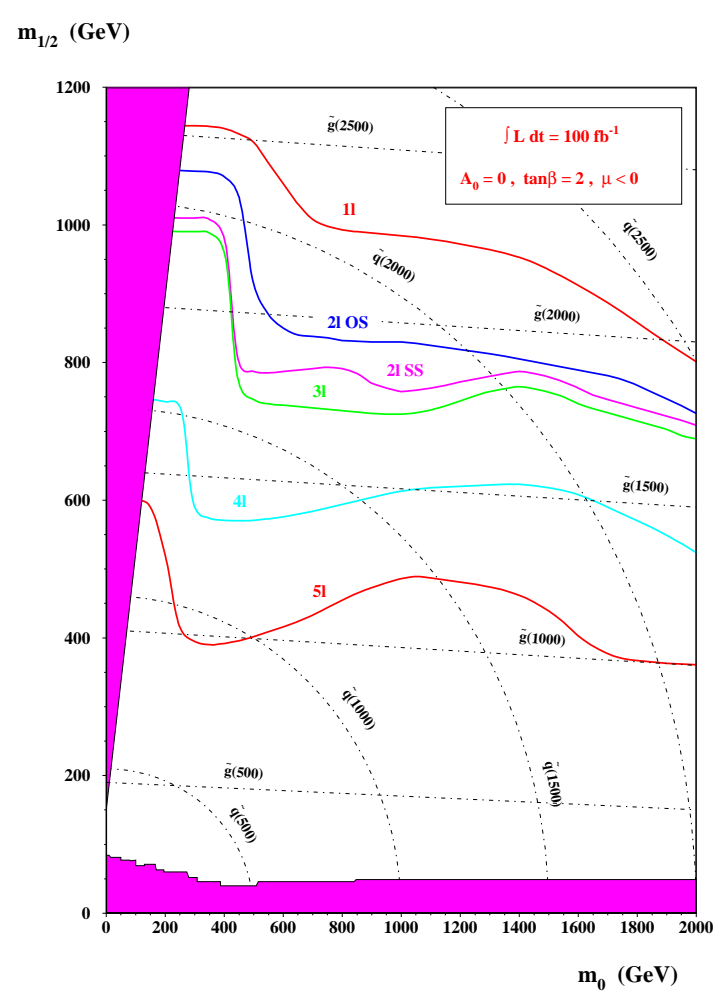

FIGURE 10. The region of the $\left(m_{0}, m_{1 / 2}\right)$ plane accessible to sparticle searches at the LHC $[22]$.

It has been suggested [52] that there may be an excess of cosmic-ray positrons at energies above $1 \mathrm{GeV}$, as seen in Fig. 13, although the uncertainties in the standard leaky-box model prevent any definite conclusion at this stage. AMS has reported an excess of positrons at lower energies: $E \lesssim 1 \mathrm{GeV}$, but it seems unlikely that these have an exotic origin. It may in the future be able to contribute to the clarification of the possible excess at higher energies.

Data from EGRET on $\gamma$ rays above $100 \mathrm{MeV}$ have been interpreted [53] as containing an excess from directions centred around the galactic centre. These could well be due to some unresolved astrophysical sources or some diffuse mechanism such as inverse Compton scattering. If due to dark matter annihilation, they would require $\left\langle\sigma v>\sim\left(10^{-24}\right.\right.$ to $\left.10^{-25}\right) \mathrm{cm}^{2}$ if the dark matter particles are not clumped. This range is above that allowed for supersymmetric dark matter: $<\sigma v>\sim 3 \times$ $10^{-27} /\left(\Omega h^{2}\right) \mathrm{cm}^{2}$. Therefore it has been suggested [54] that the dark matter may be clumped, as in some models of structure formation. A phenomenological approach to this possibility is to calculate the maximal clumpiness enhancement allowed for any supersymmetric model, taking into account the experimental upper limits on, 


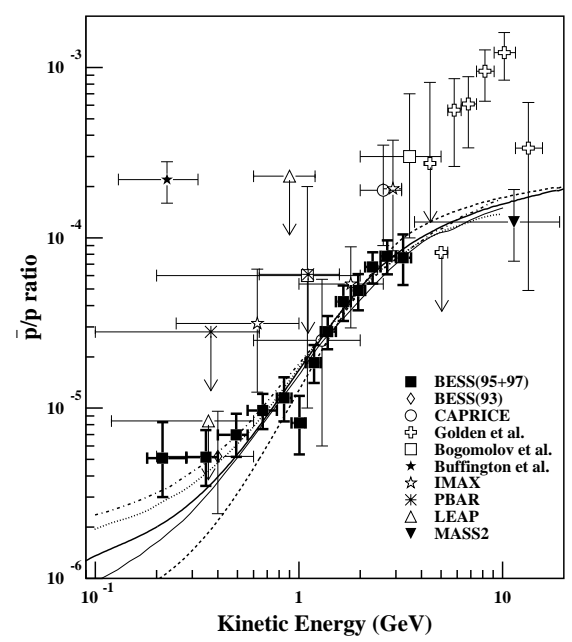

FIGURE 11. Data on $\bar{p}$ 's in the cosmic rays. The recent BESS data [49] agree with calculations based on secondary $\bar{p}$ production by primary matter cosmic rays.

e.g., $\bar{p}$ annihilation products, and then calculate the maximum possible $\gamma$ flux. Some representative calculations [54] are shown in Fig. 14: we see that the $\gamma$ fluxes could in principle be orders of magnitude larger than those detectable by GLAST.

\section{B Annihilation in the Sun or Earth}

A dark matter passing through the Sun (Earth) may scatter on some nucleus inside, losing source recoil energy which may convert its orbit from hyperbolic to elliptical, with perhelion (perigee) below the surface. Then it will scatter repeatedly, eventually settling into a quasi-thermal distribution beneath the surface. This population of relic particles is controlled by annihilation: $\chi \chi \rightarrow \ell^{+} \ell^{-}, \bar{q} q$, yielding as observable products energetic neutrinos: $E_{\nu} \gtrsim 1 \mathrm{GeV}$. These may be detected either directly in an underground detector, or indirectly via $\mu$ 's produced in material surrounding the detector.

As seen in Fig. 15 [55], a $1 \mathrm{~km}^{2}$ muon detector would be able to detect quite a number of supersymmetric models that do not produce detectable cosmic-ray $\bar{p}$ fluxes, via either the solar or subterranean cosmic rays they produce. It has recently been pointed out [56] that there could be an enhancement of relic annihilations in the Earth due to a solar-system population of relic particles that is augmented by Jupiter's gravitational field, so the prospects may be even brighter than indicated in Fig. 15. 


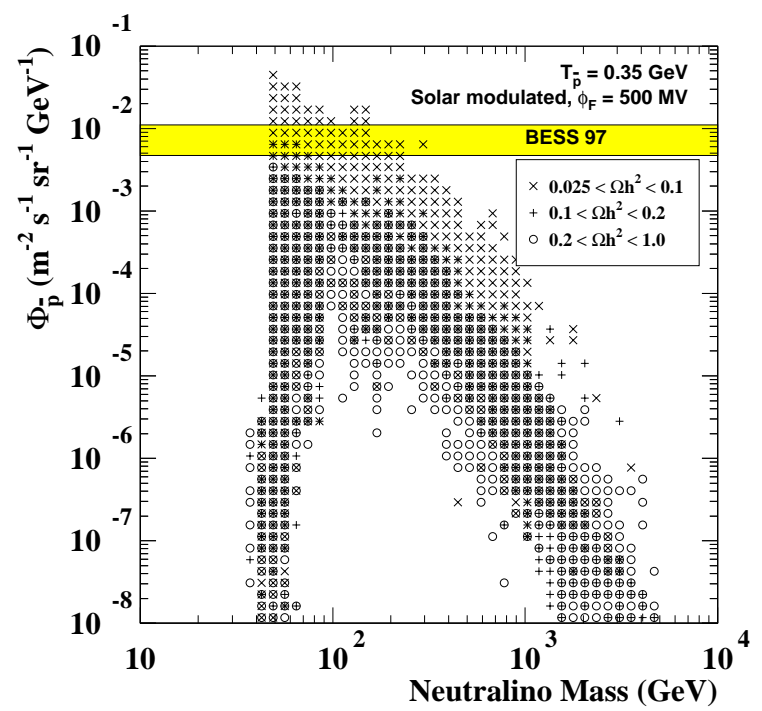

FIGURE 12. The fluxes of cosmic-ray $\bar{p}$ 's predicted in a sampling of superymmetric models [51] confronted with the BESS data [49].

\section{Direct Detection of Dark Matter}

Many experiments around the world are looking for the recoil energy deposited in low-background underground detectors by relic scattering on nuclei. The typical recoil energy deposited is $\mathcal{O}\left(m_{\chi} v^{2} / 2\right) \sim$ tens of $\mathrm{keV}$. The interaction may be mediated by squark, $Z$ and Higgs exchanges, which contribute both spin-dependent and spin-independent matrix elements. The former are related to the quark contributions to the proton spin $(\Delta q)$ and the latter to the quark contributions to the proton mass. The time-dependent contributions are important for some light nuclei such as Fluorine, where they can be calculated quite reliably. However, the spin-independent contributions are coherent and more important for heavier nuclei [57].

Figure 16 shows the upper limit for scattering on an individual nucleon, together with the region of parameter space that could not be excluded by an experiment searching for an annual modulation of the detector rate in a particular range of deposited energy [58]. Although on the large side, the range of cross section in this non-excluded domain is not inconsistent with some model calculations. Future experiments will be able to improve the current upper limits shown in Fig. 16 by several orders of magnitude and explore much of the model parameter space.

What if there were a real signal in the non-excluded region in Fig. 16? It would 


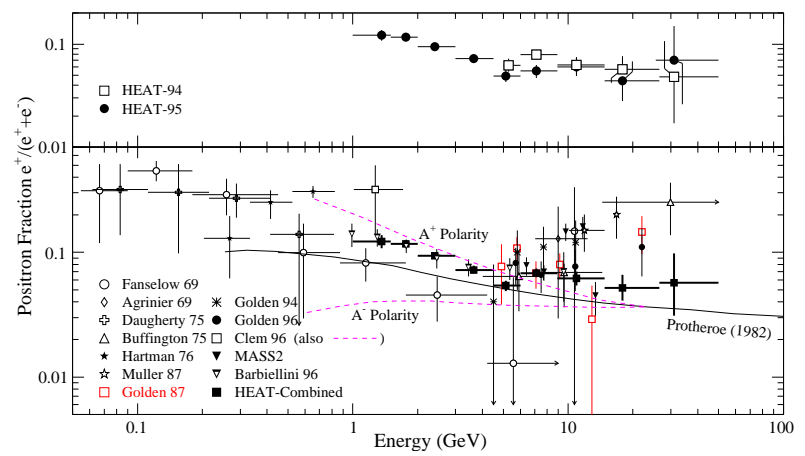

FIGURE 13. Data on the fraction of positrons in the cosmic rays [52], compared with standard leaky-box model calculations.

correspond to a supersymmetric relic weighing $\sim 50$ to $100 \mathrm{GeV}$. Such models might well produce an observable cosmic-ray $\bar{p}$ flux or $\mu$ flux from high-energy solar or subterranean neutrinos [59].

\section{Supersymmetry at the LHC}

These searches for astrophysical sparticles must compete with accelerator searches. LEP has almost completed the exploration of its available kinematic reach, and the Tevatron has a window for possible sparticle discoveries. However, the best prospects for the discovery of supersymmetry will be offered by the LHC. It will benefit from large cross sections for squark $(\tilde{q})$ and gluino $(\tilde{g})$ production, and their cascade decays into lighter sparticles offer many opportunities for distinctive signatures. As seen in Fig. 10 [47], it should be possible to detect $m_{\tilde{q} / \tilde{g}} \lesssim 2$ to $2.5 \mathrm{TeV}$, and some detailed spectroscopic measurements will be possible. The entire supersymmetric dark matter region were $\Omega_{\chi} h^{2} \lesssim 0.3$ will be covered by the LHC. So those looking for supersymmetric dark matter via cosmic rays or other astrophysical signatures should hurry up, and do their best before LHC startup in 2005 !

\section{SUPERHEAVY RELIC PARTICLES}

It has been suggested [60] that cold dark matter particles should weigh $\lesssim 1 \mathrm{TeV}$, as exemplified by the MSSM range (27). This expectation is based on the assump- 

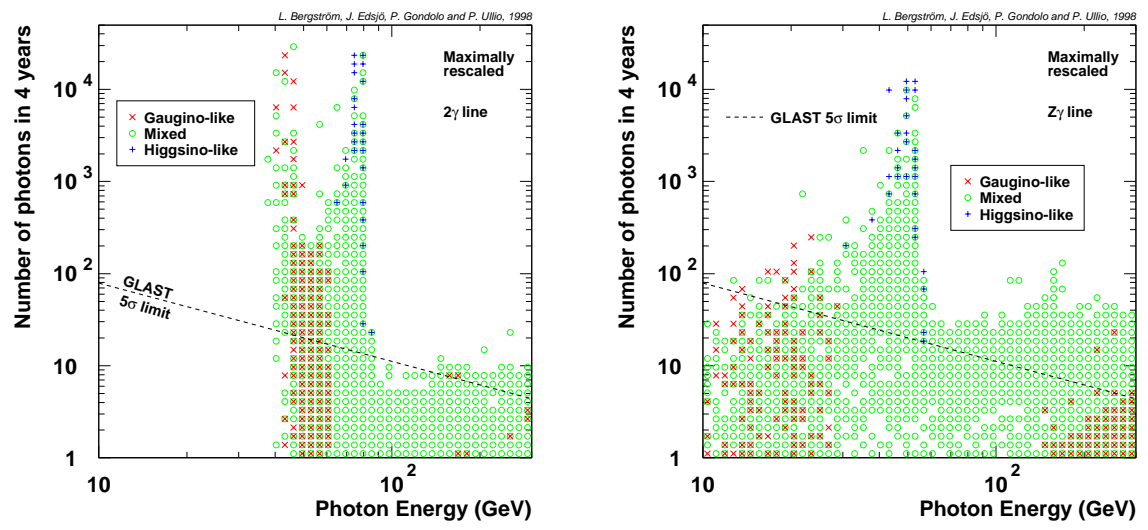

FIGURE 14. Possible fluxes of cosmic-ray $\gamma$ 's allowed if one postulates the maximal clumping of cold dark matter in the galactic halo [54], compared with the expected GLAST sensitivity.

tion that the cold dark matter particles were at one time in thermal equilibrium. However, much heavier relic particles are possible if one invokes non-thermal production mechanisms. Non-thermal decays of inflatons in conventional models of cosmological inflation could yield $\Omega_{\chi} \sim 1$ for $m_{\chi} \sim 10^{13} \mathrm{GeV}$. Preheating via the parametric resonance decay of the inflaton could even yield $\Omega_{\chi} \sim 1$ for $m_{\chi} \sim 10^{15}$ $\mathrm{GeV}$. Other possibilities include a first-order phase transition at the end of inflation, and gravitational relic production induced by the rapid change in the scale factor in the early Universe [61]. It is therefore of interest to look for possible experimental signatures of superheavy dark matter.

One such possibility is offered by ultra-high-energy cosmic rays. Those coming from distant parts of the Universe $(D \gtrsim 100 \mathrm{Mpc})$ are expected to be cut off at an energy $E \lesssim 5 \times 10^{19} \mathrm{GeV}$, because of the reaction $p+\gamma_{C M B R} \rightarrow \Delta^{+}[62]$. However, as discussed extensively here, no such Greisen-Zatsepin-Kuzmin cut-off is seen in the data [63] The ultra-high-energy cosmic rays must originate nearby, and (unless the intergalactic magnetic field is unexpectedly high [64]) should point back to any point-like sources such as AGNs [65]. However, no such discrete sources have been identified as yet.

Could the ultra-high-energy cosmic rays be due to the decays of superheavy relic particles? These should be clustered in galactic haloes (including our own), and hence give an anisotropic flux [66], but there would be no obvious point sources. There have been some reports of anisotropies in high-energy cosmic rays, but it is not clear whether they could originate in superheavy relic decays.

We analyzed [67] possible superheavy relic candidates in string [68] and/or $M$ theory. One expects Kaluza-Klein states when six excess dimensions are compact- 

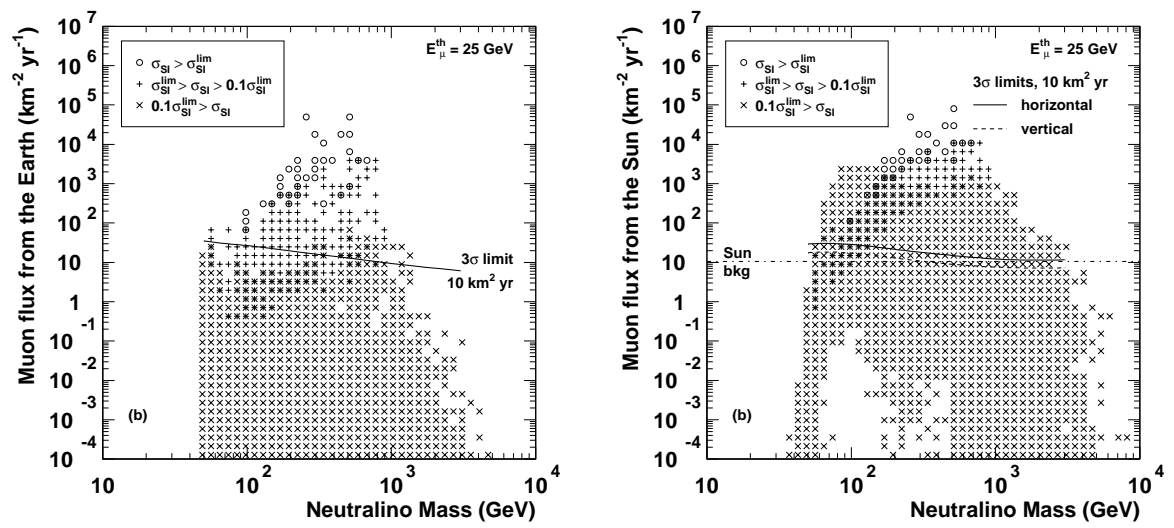

FIGURE 15. The fluxes of high-energy $\mu$ 's due to the interactions of $\nu$ 's produced by relic annihilations inside the Sun or Earth, as produced by a sampling of supersymmetric models [55], compared with the expected sensitivity of a $1 \mathrm{~km}^{2}$ detector.

ified: $10 \rightarrow 4$ or $11 \rightarrow 5$, which we call hexons. However, these are expected to weigh $\gtrsim 10^{16} \mathrm{GeV}$, which may be too heavy, and there is no particular reason to expect hexons to be metastable. In $M$ theory, one expects massive states associated with a further compactification: $5 \rightarrow 4$ dimensions, which we call pentons. Their mass could be $\sim 10^{13} \mathrm{GeV}$, which would be suitable, but there is again no good reason to expect them to be metastable. We are left with bound states from the hidden sector of string/ $M$ theory, which we call cryptons [68]. These could also have masses $\sim 10^{13} \mathrm{GeV}$, and might be metastable for much the same reason as the proton in a GUT, decaying via higher-dimensional multiparticle operators.

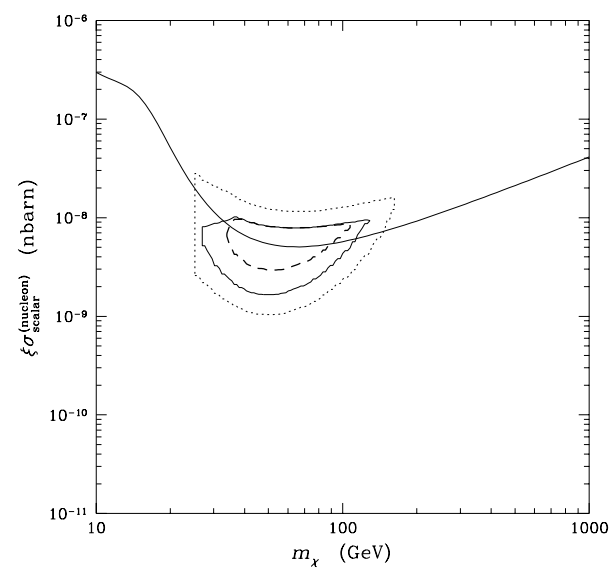

FIGURE 16. Upper limit (solid line) and regions (dotted, solid and dashed lines) not excluded by the DAMA [58] search for an annual modulation effect. 
For example, in a flipped $S U(5)$ model we have a hidden-sector $S U(4) \times S O(10)$ gauge group, and the former factor confines four-constituent states which we call tetrons. Initial studies $[68,67]$ indicate that the lightest of these might well have a lifetime $\gtrsim 10^{17} y$, which would be suitable for the decays of superheavy dark matter particles. Detailed simulations have been made of the spectra of particles produced by the fragmentation of their decay products [69,70], and the ultra-highenergy cosmic-ray data are consistent with the decays of superheavy relics weighing $\sim 10^{12} \mathrm{GeV}$, as seen in Fig. 17 [70]. Issues to be resolved here include the roles of

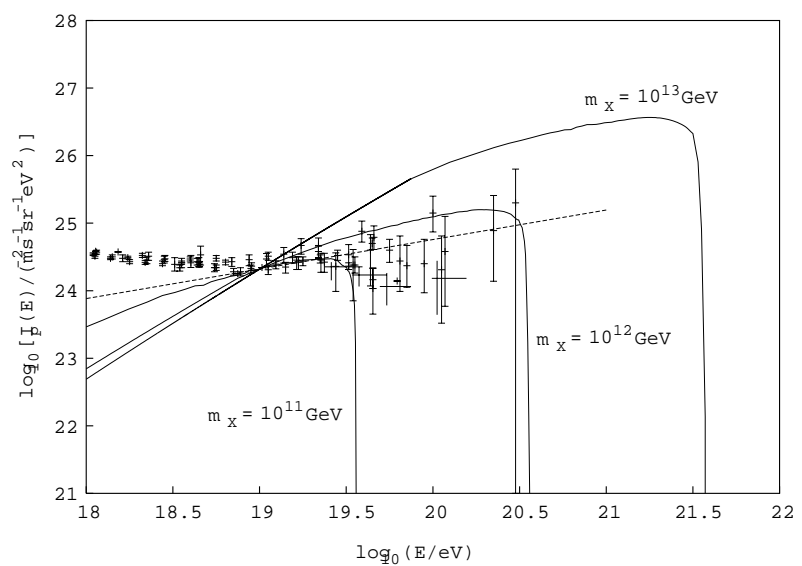

FIGURE 17. The ultra-high energy cosmic ray flux compared with a model calculation based on the decays of superheavy relic particles [70].

supersymmetric particles in the fragmentation cascades, and the relative fluxes of $\gamma, \nu$ and $p$ among the ultra-high-energy cosmic rays.

\section{VACUUM ENERGY}

As mentioned in Section 1, data on large-scale structure [6] and high-redshift supernovae [7] have recently converged on the suggestion that the energy of the vacuum may be non-zero, as seen in Figs. 1, 3. In my view, this represents a wonderful opportunity for theoretical physics: a number to be calculated in the Theory of Everything including quantum gravity. The possibility that the vacuum energy may be non-zero may even appear more natural than a zero value, since there is no obvious symmetry or other reason known why it should vanish.

In the above paragraph, I have used the term vacuum energy rather than cosmological constant, because it may not actually be constant. This option has been termed quintessence in [71], which discusses a classical scalar-field model that is not strongly motivated by the Standard Model, supersymmetry or GUTs, though 
something similar might emerge from string theory. I prefer to think that a varying vacuum energy might emerge from a quantum theory of gravity, as the vacuum relaxes towards an asymptotical value (zero?) in an infinitely large and old Universe. We have recently given [72] an example of one such possible effect which yields a contribution to the vacuum energy that decreases as $1 / t^{2}$. This is compatible with the high-redshift supernova data, and one may hope that these could eventually discriminate between such a possibility and a true cosmological constant.

\section{REFERENCES}

1. K.A. Olive, Phys. Rept. 190 (1990) 307.

2. A. Linde, Phys.Rev. D59 (1999) 023503.

3. For a recent compilation, see: M. Tegmark, http://www.sns.ias.edu/ max/r_frames.html.

4. N. Bahcall, J.P. Ostriker, S. Perlmutter and P.J. Steinhardt, Science 284 (1999) 1481.

5. D. Tytler, S. Burles, L.-M. Wu, X.-M. Fan, A. Wolfe and B.D. Savage, astro-ph/9810217.

6. N.A. Bahcall, astro-ph/9901076.

7. A.G. Riess et al., Astron. J. 116 (1998) 1009;

S. Perlmutter et al., astro-ph/9812133.

8. However, combinations of structure-formation and cosmic microwave-background measurements may be sensitive to smaller neutrino masses: W. Hu, D.J. Eisenstein, M. Tegmark and M. White, Phys. Rev. D59 (1999) 023512.

9. Particle Data Group, Particle Data Group, C. Caso et al., Eur. Phys. J. C3 (1998) 1.

10. R.D. Peccei, hep-ph/9906509, to be published in the proceedings of 8 th Mexican School of Particles and Fields (VIII-EMPC), Oaxaca de Juarez, Mexico, 20-28 Nov 1998.

11. R. Barbieri, J. Ellis and M.K. Gaillard, Phys. Lett. 90B (1980) 249.

12. M. Gell-Mann, P. Ramond and R. Slansky, Proceedings of the Stony Brook Supergravity Workshop, New York, 1979, eds. P. Van Nieuwenhuizen and D. Freedman (North-Holland, Amsterdam); T. Yanagida, Proceedings of the Workshop on Unified Theories and Baryon Number in the Universe, Tsukuba, Japan 1979, eds. A. Sawada and A. Sugamoto, KEK Report No. 79-18.

13. For an update, see: J. Bahcall, http://www.sns.ias.edu/ jnb/.

14. The literature on the subject is vast. For introductions, see: C. D. Froggatt and H. B. Nielsen, Nucl. Phys. B147 (1979) 277; H. Fritzsch, Phys. Lett. 70B (1977) 436; B73 (1978) 317; Nucl. Phys. B155 (1979) 189; J. Harvey, P. Ramond and D. Reiss, Phys. Lett. B92 (1980) 309; S. Dimopoulos, L. J. Hall and S. Raby, Phys. Rev. Lett. 68 (1992) 1984; C. Wetterich, Nucl. Phys. B261 (1985) 461; L. Ibanez and G.G. Ross, Phys. Lett. B332 (1994) 100; G.K. Leontaris and D.V. Nanopoulos, Phys. Lett. B212 (1988) 327; Y.Achiman and T. Greiner, Phys. Lett. B329 (1994) 33; Y. Grossman and Y. Nir, Nucl. Phys. B448 (1995) 30; H. Dreiner et al., Nucl. 
Phys. B436 (1995) 461; P. Binétruy, S. Lavignac and P. Ramond, Nucl. Phys. B477 (1996) 353; G.K. Leontaris, et al., Phys. Rev. D 53 (1996) 6381; P. Binétruy et al., Nucl. Phys. B496 (1997) 3; S. Lola and J.D. Vergados, Progr. Part. Nucl. Phys. 40 (1998) 71.

15. LEP Electroweak Working Group, CERN preprint EP/99-15; updates may be found at http://www. cern.ch/LEPEWWG/Welcome.html.

16. For one particular take on this, see: J. Ellis, G.K. Leontaris, S. Lola and D.V. Nanopoulos, Eur. Phys. J. C9 (1999) 389; for a recent review, see: G. Altarelli and F. Feruglio, hep-ph/9905536.

17. J. Ellis and S. Lola, Phys. Lett. B458 (1999) 310.

18. L. Baudis et al., Phys. Rev. Lett. 83 (1999) 41.

19. M. Apollonio et al., Chooz Collaboration, Phys. Lett. B338 (1998) 383 and hepex/9907037.

20. J.N. Bahcall, P.I. Krastev and A.Y. Smirnov, Phys. Rev. D60 (1999) 093001.

21. A.K. Mann, talk at the 19th International Symposium on Lepton and Photon Interactions at High Energies, Stanford, August 1999, available via: http://lp99.slac.stanford.edu/.

22. J.A. Casas, J.R. Espinosa, A. Ibarra and I. Navarro, Nucl. Phys. B556 (1999) 3, hep-ph/9905381, JHEP 9909 (1999) 015, and hep-ph/9910420.

23. R. Barbieri, L.J. Hall, G.L. Kane and G.G. Ross, hep-ph/9901228.

24. L. Maiani, Proc. Summer School on Particle Physics, Gif-sur-Yvette, 1979 (IN2P3, Paris, 1980) p. 3;

G 't Hooft, in: G' 't Hooft et al., eds., Recent Developments in Field Theories (Plenum Press, New York, 1980);

E. Witten, Nucl. Phys. B188 (1981) 513;

R.K. Kaul, Phys. Lett. 109B (1982) 19.

25. For a review, see: E. Farhi and L. Susskind, Phys. Rep. 74C (1981) 277;

S. Dimopoulos and L. Susskind, Nucl. Phys. B155 (1979) 237;

E. Eichten and K. Lane, Phys. Lett. B90 (1980) 125;

M.E. Peskin and T. Takeuchi, Phys. Rev. D46 (1992) 381;

G. Altarelli, R. Barbieri and S. Jadach, Nucl. Phys. B369 (1992) 3.

26. P. Fayet and S. Ferrara, Phys. Rep. 32 (1977) 251.

27. H.E. Haber and G.L. Kane, Phys. Rep. 117 (1985) 75.

28. H. Dreiner, hep-ph/9707435.

29. See, e.g., C. Kounnas, A.B. Lahanas, D.V. Nanopoulos and M. Quirós, Phys. Lett. 132B (1983) 95.

30. Y. Okada, M. Yamaguchi and T. Yanagida, Progr. Theor. Phys. 85 (1991) 1;

J. Ellis, G. Ridolfi and F. Zwirner, Phys. Lett. B257 (1991) 83, Phys. Lett. B262 (1991) 477;

H.E. Haber and R. Hempfling, Phys. Rev. Lett. 66 (1991) 1815;

R. Barbieri, M. Frigeni and F. Caravaglios, Phys. Lett. B258 (1991) 167;

Y. Okada, M. Yamaguchi and T. Yanagida, Phys. Lett. B262 (1991) 54.

31. J. Ellis, S. Kelley and D.V. Nanopoulos, Phys. Lett. B249 (1990) 441 and Phys. Lett. B260 (1991) 131;

U. Amaldi, W. de Boer and H. Furstenau, Phys. Lett. B260 (1991) 447; 
P. Langacker and M. Luo, Phys. Rev. D44 (1991) 817.

32. J. Ellis, J.S. Hagelin, D.V. Nanopoulos, K.A. Olive and M. Srednicki, Nucl. Phys. B238 (1984) 453.

33. For a recent review, see: K.A. Olive, hep-ph/9911307.

34. J. Rich, M. Spiro and J. Lloyd-Owen, Phys. Rep. 151 (1987) 239;

P.F. Smith, Contemp. Phys. 29 (1998) 159;

T.K. Hemmick et al., Phys. Rev. D41 (1990) 2074.

35. R.N. Mohapatra and S. Nussinov, Phys. Rev. D57 (1998) 1940.

36. J. Ellis, T. Falk, K. Olive and M. Schmitt, Phys. Lett. B388 (1996) 97 and Phys. Lett. B413 (1997) 355.

37. H.V. Klapdor-Kleingrothaus and Y. Ramachers, Eur. Phys. J. A3 (1998) 85.

38. G. Giudice and R. Rattazzi, hep-ph/9801271.

39. J. Ellis, T. Falk, K. Olive and M. Schmitt, Phys. Lett. B413 (1997) 355.

40. LEP Experiments Committee meeting, Nov. 9th, 1999, http://delphiwww.cern.ch/ offline/physics_links/lepc.html.

41. J. Ellis, K. Enqvist, D.V. Nanopoulos and F. Zwirner, Mod. Phys. Lett. A1 (1986) 57 ;

G.F. Giudice and R. Barbieri, Nucl. Phys. B306 (1988) 63.

42. P.H. Chankowski, J. Ellis and S. Pokorski, Phys. Lett. B423 (1998) 327;

P.H. Chankowski, J. Ellis, M. Olechowski and S. Pokorski, Nucl. Phys. B544 (1999) 39.

43. P.H. Chankowski, J. Ellis, K.A. Olive and S. Pokorski, Phys. Lett. B452 (1999) 28.

44. S. Abel and T. Falk, Phys. Lett. B444 (1998) 427.

45. K.A. Olive and M. Srednicki, Phys. Lett. B230 (1989) 78 and Nucl. Phys. B355 (1991) 208;

K. Griest, M. Kamionkowski and M.S. Turner, Phys. Rev. D41 (1990) 3565.

46. J. Ellis, T. Falk and K.A. Olive, Phys. Lett. B444 (1998) 367;

J. Ellis, T. Falk, K.A. Olive and M. Srednicki, hep-ph/9905481.

47. S. Abdullin and F. Charles, Nucl. Phys. B547 (1999) 60.

48. J. Silk and M. Srednicki, Phys. Rev. Lett. 53 (1984) 624.

49. S. Orito et al., BESS collaboration, astro-ph/9906426.

50. R. Battiston, for the AMS collaboration, astro-ph/9907152.

51. L. Bergstrom, J. Edsjo and P. Ullio, astro-ph/9906034, these Proceedings, Vol. 2, p.285.

52. S. Coutu et al., HEAT collaboration, Astropart. Phys. 11 (1999) 429.

53. D.D. Dixon et al., New Astron. 3 (1998) 539.

54. L. Bergstrom, J. Edsjo, P. Gondolo and P. Ullio, Phys. Rev. D59 (1999) 043506

55. L. Bergstrom, J. Edsjo and P. Gondolo, astro-ph/9906033, these Proceedings, Vol. 2, p.281.

56. T. Damour and L.M. Krauss, Phys. Rev. D59 (1999) 063509;

L. Bergstrom, T. Damour, J. Edsjo, L.M. Krauss and P. Ullio, JHEP 08 (1999) 010; see however: A. Gould and S.M. Alam, astro-ph/9911288.

57. K. Griest, G. Jungman and M. Kamionkowski, Phys. Rept. 267 (1996) 195.

58. R. Bernabei et al., DAMA Collaboration, Phys. Lett. B450 (1999) 448.

59. A. Bottino and N. Fornengo, hep-ph/9904469. 
60. S. Dimopoulos, Phys. Lett. B246 (1990) 347.

61. E.W. Kolb, D.J.H. Chung and A. Riotto, hep-ph/9810361 and references therein.

62. K. Greisen, Phys. Rev. Lett. 16 (1966) 748;

G.T. Zatsepin and V.A. Kuzmin, Pis'ma Zh. Eksp. Teor. Fiz. 4 (1966) 114.

63. M. Takeda et al., Phys. Rev. Lett. 81 (1998) 1163 and references therein.

64. E. Ahn, G. Medina-Tanco, P.L. Biermann and T. Stanev, astro-ph/9911123.

65. The apparent excess of ultra-high-energy cosmic rays is significantly reduced if their sources follow the known inhomogeneous distribution of matter in the nearby Universe: G. Medina Tanco, astro-ph/9905239, these Proceedings, Vol. 4, p.346.

66. V. Berezinsky and A. Mikhailov, Phys. Lett. B449 (1999) 237;

G.A. Medina Tanco and A.A. Watson, Astropart. Phys. 12 (1999) 25.

67. K. Benakli, J. Ellis and D.V. Nanopoulos, Phys. Rev. D59 (1999) 047301.

68. J. Ellis, J. Lopez and D.V. Nanopoulos, Phys. Lett. B247 (1990) 257;

J. Ellis, G. Gelmini, J. Lopez, D.V. Nanopoulos and S. Sarkar, Nucl. Phys. B373 (1992) 399.

69. V. Berezinsky, M. Kachelriess and A. Vilenkin, Phys. Rev. Lett. 79 (1997) 4302;

V. Berezinsky and M. Kachelriess, Phys. Lett. B434 (1998) 61.

70. M. Birkel and S. Sarkar, Astropart. Phys. 9 (1998) 297.

71. I. Zlatev, L.M. Wang, P.J. Steinhardt, Phys. Rev. Lett. 82 (1999) 896.

72. J. Ellis, N.E. Mavromatos and D.V. Nanopoulos, gr-qc/9810086. 